\title{
Sex differences in the ability of corticostriatal oscillations to predict rodent alcohol consumption
}

\author{
Angela M. Henricks ${ }^{1 * \dagger} \mathbb{D}$, Emily D. K. Sullivan ${ }^{1 \dagger}$, Lucas L. Dwiel ${ }^{1}$, Karina M. Keus ${ }^{2}$, Ethan D. Adner ${ }^{2}$,
} Alan I. Green ${ }^{1,2,3}$ and Wilder T. Doucette ${ }^{1,2,3}$

\begin{abstract}
Background: Although male and female rats differ in their patterns of alcohol use, little is known regarding the neural circuit activity that underlies these differences in behavior. The current study used a machine learning approach to characterize sex differences in local field potential (LFP) oscillations that may relate to sex differences in alcohol-drinking behavior.

Methods: LFP oscillations were recorded from the nucleus accumbens shell and the rodent medial prefrontal cortex of adult male and female Sprague-Dawley rats. Recordings occurred before rats were exposed to alcohol ( $n=10 /$ sex $\times 2$ recordings/rat) and during sessions of limited access to alcohol ( $n=5 / \mathrm{sex} \times 5$ recordings/rat). Oscillations were also recorded from each female rat in each phase of estrous prior to alcohol exposure. Using machine learning, we built predictive models with oscillation data to classify rats based on: (1) biological sex, (2) phase of estrous, and (3) alcohol intake levels. We evaluated model performance from real data by comparing it to the performance of models built and tested on permutations of the data.

Results: Our data demonstrate that corticostriatal oscillations were able to predict alcohol intake levels in males $(p<0.01)$, but not in females $(p=0.45)$. The accuracies of models predicting biological sex and phase of estrous were related to fluctuations observed in alcohol drinking levels; females in diestrus drank more alcohol than males $(p=0.052)$, and the male vs. diestrus female model had the highest accuracy $(71.01 \%)$ compared to chance estimates. Conversely, females in estrus drank very similar amounts of alcohol to males $(p=0.702)$, and the male vs. estrus female model had the lowest accuracy (56.14\%) compared to chance estimates.

Conclusions: The current data demonstrate that oscillations recorded from corticostriatal circuits contain significant information regarding alcohol drinking in males, but not alcohol drinking in females. Future work will focus on identifying where to record LFP oscillations in order to predict alcohol drinking in females, which may help elucidate sex-specific neural targets for future therapeutic development.
\end{abstract}

Keywords: Alcohol, Local field potentials, Sex differences, Corticostriatal circuits, Machine learning

\footnotetext{
* Correspondence: Angela.m.henricks@dartmouth.edu

${ }^{\dagger}$ Angela M. Henricks and Emily D. K. Sullivan contributed equally to this work.

${ }^{1}$ Department of Psychiatry, Geisel School of Medicine at Dartmouth, Medical

Center Drive, Lebanon NH 03756, USA

Full list of author information is available at the end of the article
}

(c) The Author(s). 2019 Open Access This article is distributed under the terms of the Creative Commons Attribution 4.0 International License (http://creativecommons.org/licenses/by/4.0/), which permits unrestricted use, distribution, and reproduction in any medium, provided you give appropriate credit to the original author(s) and the source, provide a link to the Creative Commons license, and indicate if changes were made. The Creative Commons Public Domain Dedication waiver (http://creativecommons.org/publicdomain/zero/1.0/) applies to the data made available in this article, unless otherwise stated. 


\section{Background}

Alcohol use contributes to 5.1\% of the global disease burden, accounting for $5 \%$ of all deaths in men and $1 \%$ of all deaths in women in the USA alone [1-3]. While historically men drink more alcohol than women, this gender gap is closing [4], and women tend to escalate to alcohol dependence more rapidly than men $[2,5]$. Though these sex differences partly arise from sociocultural factors, there are known sex differences in the activity of brain regions that underlie substance use behavior $[5,6]$. However, the specific neurobiological underpinnings contributing to sex differences in alcohol drinking are poorly understood, limiting the development of more efficacious, targeted therapies for problematic alcohol use.

One barrier to the development of better therapies for excessive alcohol use is the fact that the majority of preclinical neuroscience studies have used only male animals $[7,8]$. However, the available behavioral data in rodent models of alcohol drinking demonstrate that female rats, in a non-dependent state, drink more alcohol and show greater alcohol preference than male rats [9], as well as display heightened sensitivity to the rewarding effects of alcohol compared to males [10]. The behavioral differences between females and males are biological in nature as neonatal masculinization of females reduces alcohol intake compared with intact female rats, resulting in patterns of drinking similar to those displayed by males [11]. In a similar study, intact female rats showed a heightened reward response to alcohol than either males or ovariectomized females, suggesting that ovarian hormones help facilitate the reinforcing properties of alcohol [10]. Ovarian hormone status has also been associated with small fluctuations in alcohol consumption in intact females [12, 13]. However, it is currently unknown whether the neural circuits that regulate alcohol consumption show sexually dimorphic activity patterns (and whether these patterns are influenced by ovarian hormone status) that may explain the sex differences in alcohol drinking behavior.

The mechanistic role of corticostriatal circuits in regulating the rewarding properties of alcohol is well characterized in male rodents [14]. In rats (and humans), the nucleus accumbens (NAc) integrates cortical inputs and indirectly sends feedback to frontal brain regions (medial prefrontal cortex in humans (mPFC); prelimbic (PL), and infralimbic (IL) cortices in rats) [15] and is particularly important in the motivating properties of abused drugs [16]. The mPFC is also activated in response to reward-related cues, and it has been suggested that deficits in the ability to inhibit responses to drugs arises from dysregulated communication between the mPFC and striatal regions [17]. Thus, we hypothesize that male and female rats might display inherent (i.e., trait-level) differences in corticostriatal circuit activity, which may be associated with sex differences in alcohol-drinking behaviors.

Activity in the corticostriatal circuit can be examined longitudinally by measuring local field potential (LFP) oscillations in awake, freely behaving rats. LFP oscillations provide a readout of electrical potential from a group of neurons that relates to individual neuronal activity, as demonstrated by neuronal phase locking and ensemble classification [18-20]. LFP oscillations recorded from reward-related regions have been shown to change during behavior [21] and reflect pharmacologic manipulation [22-24]. For instance, in male rats, lowfrequency oscillations decrease while high-frequency oscillations increase following an injection of alcohol [25]. Furthermore, low-frequency oscillations in the cortex and NAc appear to be hypoconnected in alcohol-preferring rats (sex not reported) compared to outbred rats, which was reversed by alcohol exposure [26]. LFP oscillations can therefore be a valuable readout of circuit dynamics related to alcohol-drinking behaviors (i.e., amount of alcohol consumed) in rodents.

In the current experiment, we measured corticostriatal LFP oscillations in adult male and female rats prior to and during alcohol-drinking behavior. Using an unbiased machine learning approach, we aimed to determine whether LFPs recorded from corticostriatal circuits contained information regarding: (1) biological sex, (2) ovarian hormone status, and (3) the amount of alcohol consumed during an alcohol drinking session. We hypothesized that sex differences in inherent corticostriatal circuit activity might be related to sex differences in alcohol-drinking behavior.

\section{Methods}

\section{Subjects and housing}

Male and female Sprague-Dawley rats $(n=10 /$ sex $)$ were purchased from Charles River (Wilmington, MA, USA) and arrived on postnatal day 60. All animals were housed individually on a reverse 12-h light cycle with ad libitum access to food and water. All experiments were carried out in accordance with the National Institute of Health Guide for the Care and Use of Laboratory Animals (NIH Publications No. 80-23) and were approved by the Institutional Animal Care and Use Committee of Dartmouth College.

\section{Electrode construction and implantation}

Electrodes were designed and constructed in-house and were similar to those used in our previous publication [27]. Animals were anesthetized with isoflurane gas (4\% induction, $2 \%$ maintenance) and secured into a stereotaxic frame. Custom electrodes were implanted bilaterally targeting the NAc shell (NAcSh; from bregma: DV $-8 \mathrm{~mm}$; AP +1.2 $\mathrm{mm} ; \mathrm{ML} \pm 1.0 \mathrm{~mm}$ ) and PL/IL junction of the $\mathrm{mPFC}$ (from bregma: DV $-5 \mathrm{~mm}$; AP $+3.7 \mathrm{~mm} ; \mathrm{ML} \pm 0.75 \mathrm{~mm}$ ). The NAcSh was targeted based on previous work demonstrating that deep brain stimulation of the NAcSh could reduce alcohol intake in male $\mathrm{P}$ rats and high-alcohol drinking 
outbred rats $[28,29]$. Thus, we hypothesized that LFPs recorded from the NAcSh would contain information regarding alcohol intake in rats. Four stainless steel skull screws were placed around the electrode site and dental cement (Dentsply, York, PA, USA) was applied to secure the electrodes in place.

Recording and processing local field potential oscillations LFP oscillations were recorded in sound-attenuated chambers distinct from the rats' home cages. Rats engaged in free behavior while tethered through a commutator to a Plexon data acquisition system and timesynchronized videos were recorded for each session (Plexon, Plano, TX). Noise-free data from the entire recording session were analyzed using established frequency ranges from the rodent literature (delta $(\Delta)=1-$ $4 \mathrm{~Hz}$, theta $(\theta)=5-10 \mathrm{~Hz}$, alpha $(\alpha)=11-14 \mathrm{~Hz}$, beta $(\beta)=15-30 \mathrm{~Hz}$, low gamma $(\mathrm{l} \gamma)=45-65 \mathrm{~Hz}$, and high gamma (hy) $70-90 \mathrm{~Hz}[30,31])$ and standard LFP signal processing was used to characterize the power spectral densities (PSDs) within, and coherence between brain regions for each rat using custom code written for Matlab R2017b. A fourth order Chebychev type I notch filter centered at $60 \mathrm{~Hz}$ was applied to all of the data to account for $60-\mathrm{Hz}$ line noise. The data was then downsampled by a factor of five from $2 \mathrm{kHz}$ to $400 \mathrm{~Hz}$. A threshold of $\pm 2 \mathrm{mV}$ was used to identify noise artifacts and remove data using intervals 12.5 milliseconds before and $40 \mathrm{~s}$ after the artifacts. To capture the power and coherence dynamics of the signal, we used only epochs that were at least $3 \mathrm{~s}$ long. For epochs that were longer than $3 \mathrm{~s}$, we segmented them into 3 -s sections removing the remainder to keep all of the data continuous over the same amount of time. An example trace LFP oscillation is shown in Fig. 1a.

PSDs were computed using MATLAB's pwelch function using a 1.6-s Hamming window with 50\% overlap. The PSDs for each 3-s segment were then averaged together to get a single representative PSD for the 30-min recording session. Total power $(\mathrm{dB})$ was calculated for each frequency range. To account for the $60-\mathrm{Hz}$ notch filter, power values of frequencies from 59 to $61 \mathrm{~Hz}$ were not included in the analysis. The power per frequency band was then normalized as a percent of the average total power of the signal from 1 to $90 \mathrm{~Hz}$ (beginning of $\Delta$ to end of $h \gamma$ ).

Coherence was computed using the function mscohere with a $1.3 \mathrm{~s}$ sliding Hamming window with $50 \%$ overlap. The average coherence between each pair of frequency bands from 1 to $90 \mathrm{~Hz}$ (excluding 59 to $61 \mathrm{~Hz}$ ) was used to normalize the average coherence of each frequency band within that neural site pair.

\section{Determination of estrous phase}

After each baseline recording session, estrous cycle was determined via vaginal lavage as described previously [13]. Slides were stained using thionin and the stage of estrus was assessed using an AmScope light microscope (Irvine, CA). Proestrus was characterized as $>75 \%$ of the cells in the sample being nucleated epithelial cells. Estrus was characterized as dense sheets of cornified epithelial cells, and diestrus was characterized as scattered nucleated and cornified epithelial cells, along with leukocytes (diestrus-1), or the relative lack of any cells (diestrus-2).

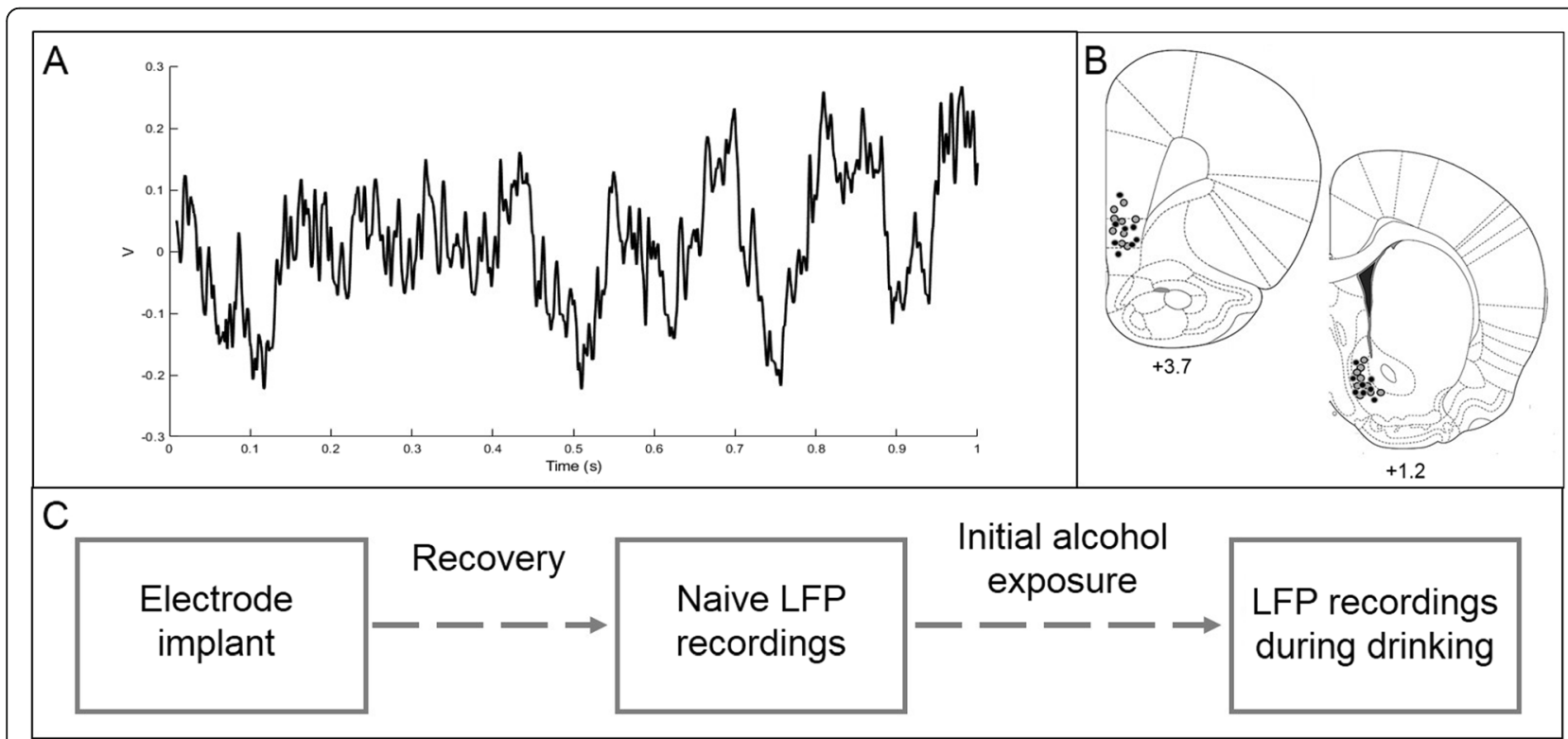

Fig. 1 A sample trace of corticostriatal oscillations used in the prediction models (a). Histology figures representing electrode placements in the NAcSh and mPFC. Males are represented by black dots and females are represented by gray dots (b). Experimental timeline (c) 


\section{Verification of electrode placement}

At the end of the experiment, rats were euthanized using $\mathrm{CO}_{2}$ gas, brains were extracted and subsequently snap frozen in 2methylbutane on dry ice. Tissue was stored at $-20^{\circ} \mathrm{C}$ prior to being sectioned at $40 \mu \mathrm{m}$ using a Leica CM1850 cryostat and stained with thionin. Electrode placement was verified using an AmScope light microscope (Irvine, CA). Figure 1b shows the electrode placements. Three animals' (two males and one female) brains were not preserved properly so we were unable to verify electrode placements in those rats. However, based on our previous experience targeting the NAcSh and mPFC with $<1 \%$ misplacement, we elected to include the data from that those animals' in this study.

\section{Experimental overview}

Following 1 week of habituation to the animal facility, rats were implanted with bilateral recording electrodes targeting corticostriatal regions. After at least 1 week of recovery, baseline LFPs were recorded in two, 30-min sessions for each male rat, and in each phase of estrous (proestrus, estrus, and diestrus) for each female rat. After baseline LFP recordings were collected, rats were allowed to drink $10 \%$ alcohol $(100 \%$ ethanol diluted with tap water) in a limited access paradigm for 9 sessions (90 min a day, MWF, in a neutral chamber) in order to introduce each rat to alcohol. Animal weights and the volume of alcohol consumed were measured following each session in order to calculate $\mathrm{g} / \mathrm{kg}$ of alcohol consumed. Next, LFP oscillations were recorded without access to alcohol for $15 \mathrm{~min}$, and then with access to alcohol for $30 \mathrm{~min}$, across five distinct sessions. It is important to note that the male rats in this study were also used for a separate study investigating the impact of deep brain stimulation on alcohol-drinking behaviors. See Fig. 1c for an experimental timeline.

\section{Statistical analysis \\ Linking corticostriatal LFPs to biological sex and phase of estrous}

In order to link corticostriatal activity to biological sex or phase of estrous we used an unbiased machine learning approach similar to what we have published previously [32, 33]. We built predictive models using corticostriatal LFPs to classify rats by biological sex and female rats by phase of estrous. Each recording session produced 60 LFP features: 24 measures of power ( 6 frequency bands $\times 4$ channels) and 36 measures of coherence ( 6 frequency bands $\times 6$ channel combinations). We used a penalized regression method (lasso) in order to capture potential combinations of LFP features that correlated with biological sex or phase of estrous. The Matlab package Glmnet [34] was used to implement the lasso using a fourfold cross-validation with 100 repetitions for each of the following models: (1) male vs. female (diestrus), (2) male vs. female (estrus), (3) male vs. female (proestrus), (4) diestrus vs. estrus, (5) diestrus vs. proestrus. and (6) estrus vs. proestrus. The accuracy of the model is reported as the average cross-validated accuracy.

\section{Permutation testing}

In order to assess the relative accuracy of the prediction models, we compared the real model performance to models built and tested on 100 different random permutations of the data. As the outcomes of these models are binary, the random permutation models should estimate chance predictions. Thus, if the real models performed better than chance, we determined that there is some information in the circuit related to our binary outcome. Because we used multiple recording sessions from the same rat as separate samples in the real model, we also evaluated models built on permutations of binary rat groupings (group permutations). This was done by keeping the LFP oscillation data together with the rat it was recorded from and shuffling the group assignment of each rat's set of recordings. Biological sex was equally represented in each group (see Fig. 2). The group permutation test thus evaluated the information contained within LFPs about all possible rat groupings. We calculated the mean accuracy and 95\% confidence intervals of cross-validated accuracy from the real, random permutation, and group permutation distributions, as well as $z$-scores comparing the real and random permutation distributions.

\section{Linking corticostriatal LFPs to alcohol intake levels}

In order to analyze the impact of hormone status on alcohol intake during the recording sessions, we used a linear mixed model because two females were lacking at least one drinking day in either estrus or proestrus. Hormone status (diestrus, proestrus, estrus, or male) was used as the fixed effect, controlling for rat identification as the random effect, to predict alcohol intake during each session.

We used a similar machine learning approach (as described above) to link corticostriatal activity to alcohol intake levels, except the outcomes were continuous (g/ $\mathrm{kg}$ of alcohol consumed by each rat across each day) rather than binary. $P$ values were calculated to determine the magnitude of the difference between the random permutation distributions and the real distributions. Additionally, if the lasso indicated that information existed in the LFP signal, we implemented exhaustive single feature regressions using each LFP predictor to determine the relative information content of each feature, as we have previously described in detail [33].

\section{Results}

The ability of corticostriatal LFPs to predict biological sex depends on female estrous phase

Models built from corticostriatal LFP features were able to outperform randomly permuted data in predicting biological sex, and the accuracy of the model performance 


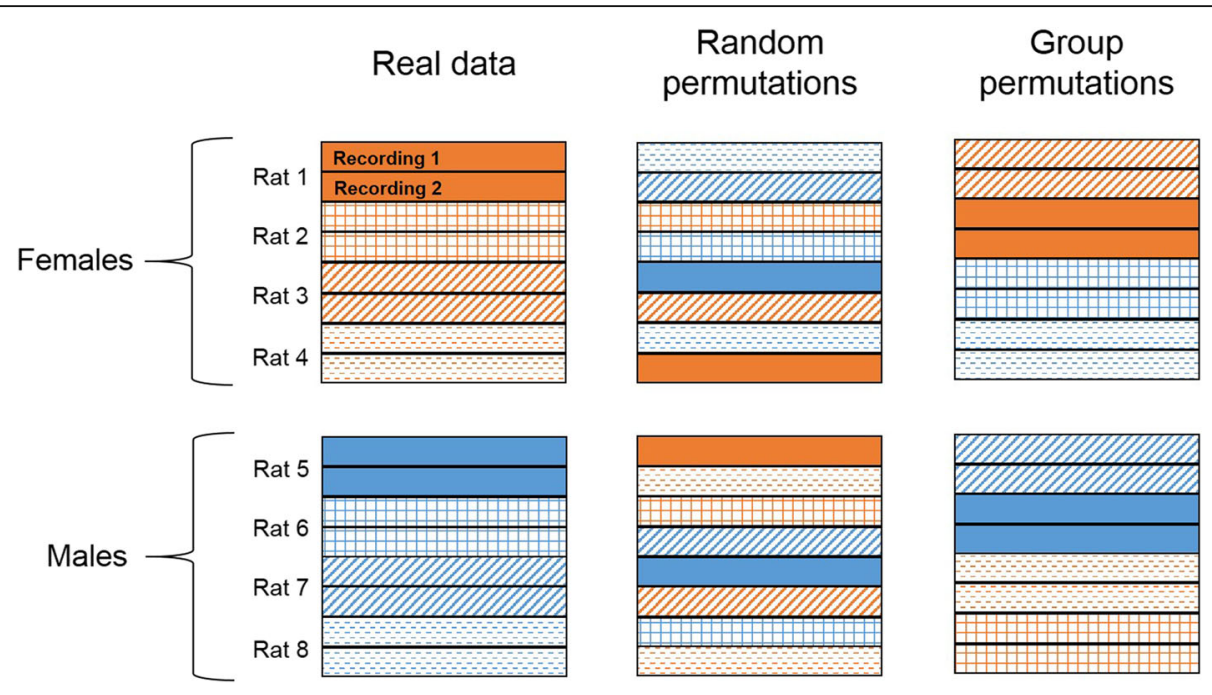

Fig. 2 Schematic representation of the permutation testing. Each set of bars represents data from one rat (if each rat has two recordings), with males in blue and females in orange. Randomly permuted models are built on 100 iterations of shuffled data. Group permutation models are built on all possible combinations of rats assigned to each group (e.g., male or female), but the two recordings from each rat are kept together and males and females are equally represented in each permutation

depended on the hormone status of the females. Models predicting males vs. females in diestrus performed with the highest average accuracy; Fig. 3 shows the predictive models for males vs. females in diestrus (random permutation $\mu=54.96 \pm 0.6 \%$, real $\mu=71.01 \pm 1 \%, z=1.71$; $3 \mathrm{~A})$, males vs. females in proestrus (random permutation $\mu=$ $43.85 \pm 0.8 \%$, real $\mu=57.7 \pm 1.5 \%, z=1.09$; 3B), and males vs. females in estrus (random permutation $\mu=48.15 \pm 0.6 \%$, real $\mu=56.1 \pm 1.3 \%, z=0.81$; 3 C). It is important to note, however, that models built on group permutations of male vs. females in diestrus performed just as well as the real models (group permutation $\mu=73.28 \pm 0.0002$ ), indicating that the magnitude of sex-based differences corticostriatal circuit activity was no greater than random groupings of rats (balanced for sex) in this sample.

For the female rats, the accuracy of models built from corticostriatal LFP features to predict phase of estrous fluctuated based on hormone status. Models predicting estrus vs. diestrus performed with the highest accuracy; Fig. 4 shows the predictive models for estrus vs. diestrus (random permutation $\mu=50.72 \pm 0.6 \%$, real $\mu=64.92 \pm$ $1.2 \%, z=1.57 ; 4 \mathrm{~A}$ ), estrus vs. proestrus (random

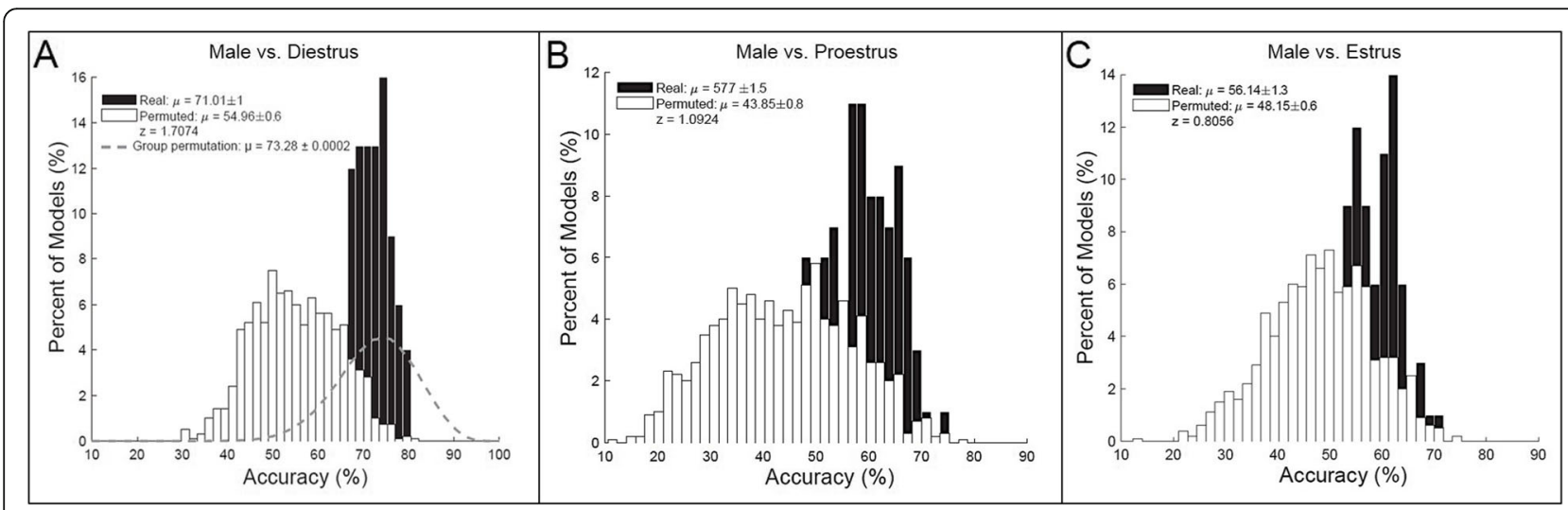

Fig. 3 Biological sex (i.e., trait-level) prediction models ( $n=10 /$ sex $\times 2$ recordings/rat). Corticostriatal LFP oscillations predicting males vs. females in diestrus (random permutation $\mu=54.96 \pm 0.6 \%$, animal permutation $\mu=73.28 \pm 0.0002 \%$; real $\mu=71.01 \pm 1 \%, z=1.71$; a), males vs. females in proestrus (random permutation $\mu=43.85 \pm 0.8 \%$, real $\mu=57.7 \pm 1.5 \%, z=1.09 ; \mathbf{b}$ ), and males vs. females in estrus (random permutation $\mu=$ $48.15 \pm 0.6 \%$, real $\mu=56.1 \pm 1.3 \%, z=0.81 ; \mathbf{c})$ 


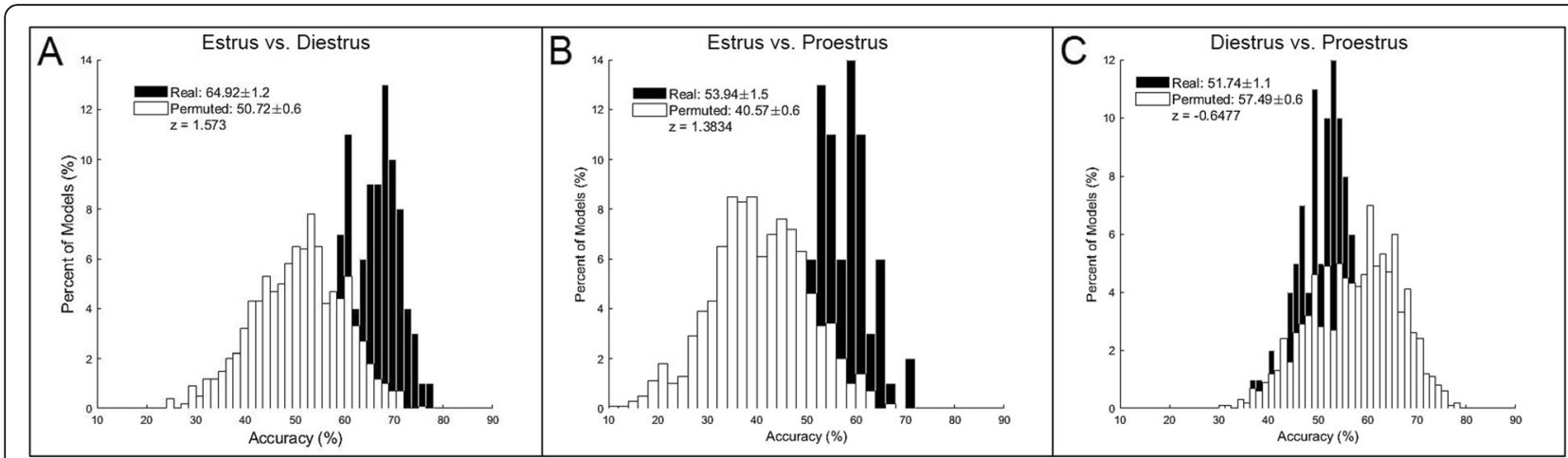

Fig. 4 Phase of estrous prediction models ( $n=10 \times 2$ recordings/phase). Corticostriatal LFP oscillations predicting estrus vs. diestrus (random permutation $\mu=50.72 \pm 0.6 \%$, real $\mu=64.92 \pm 1.2 \%, z=1.57$; a), estrus vs. proestrus (random permutation $\mu=40.97 \pm 0.6 \%$, real $\mu=53.94 \pm 1.5 \%$, $z=1.38 ; \mathbf{b}$ ), and diestrus vs. proestrus (random permutation $\mu=57.49 \pm 0.6 \%$, real $\mu=51.74 \pm 1.1 \%, z=-0.65 ; \mathbf{c}$ )

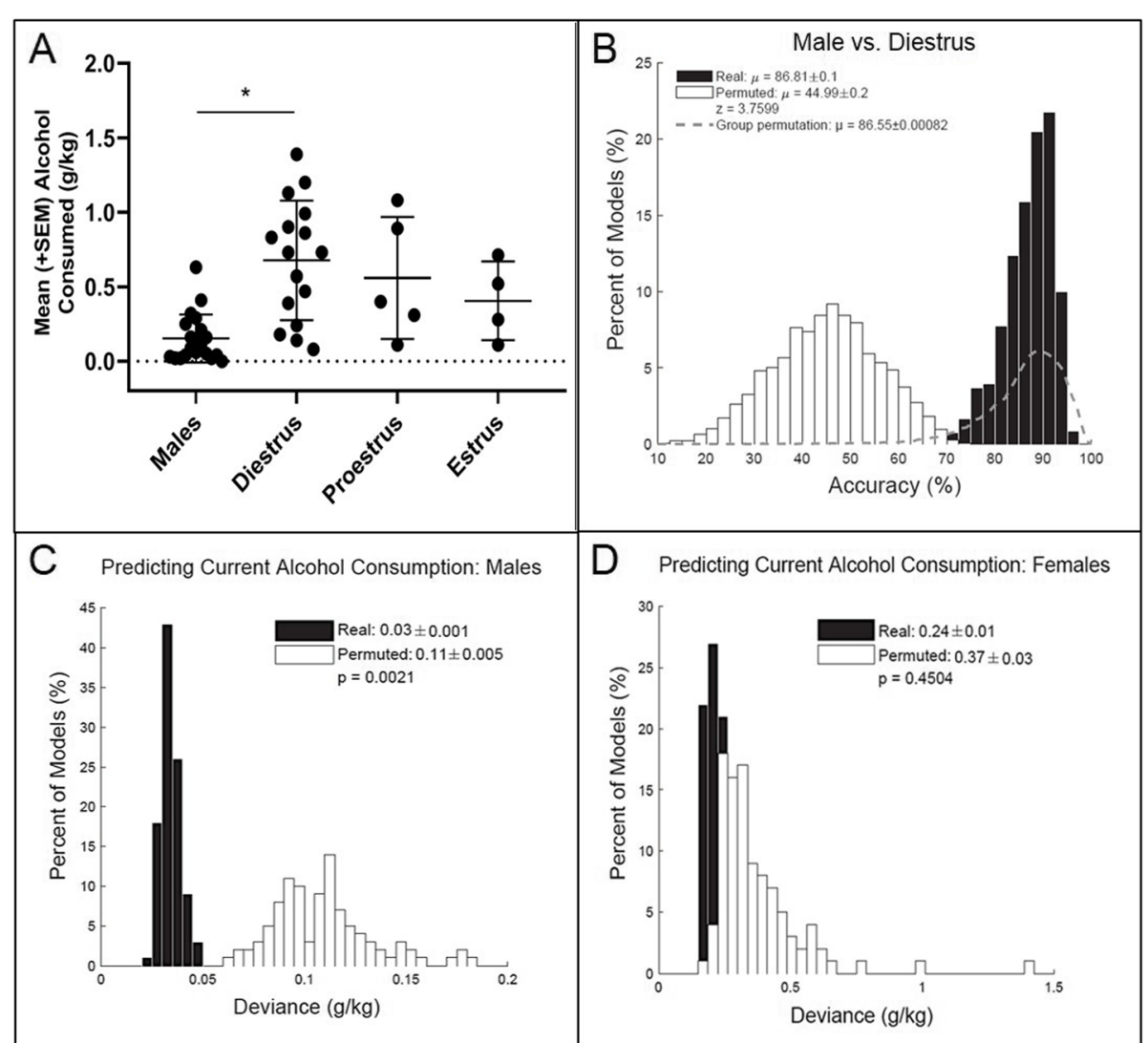

Fig. 5 Predicting alcohol intake levels $(n=5 / \mathrm{sex} \times 5$ recordings/rat). Female rats in diestrus drank more alcohol than male rats $(p=0.052 ; \mathbf{a})$. Corticostriatal LFP oscillations predicting males vs. females in diestrus during alcohol intake sessions (random permutation $\mu=44.99 \pm 0.2 \%$, animal permutation $\mu=86.55 \pm 0.0008$, real $\mu=86.81 \pm 0.01 \%, z=3.76$; b). Corticostriatal LFP oscillations predict alcohol intake levels in males (random permutation error $=0.11 \pm 0.005$, real error $=0.03 \pm 0.001, p<0.01 ; \mathbf{c}$ ), but not in females (random permutation error $=0.37 \pm 0.03$, real error $=0.24 \pm 0.01, p=0.45$; $)$ 
permutation $\mu=40.97 \pm 0.6 \%$, real $\mu=53.94 \pm 1.5 \%, z=$ $1.38 ; 4 \mathrm{~B}$ ), and diestrus vs. proestrus (random permutation $\mu=57.49 \pm 0.6 \%$, real $\mu=51.74 \pm 1.1 \%, z=-0.65 ; 4$ C).

\section{Corticostriatal LFPs predict alcohol intake levels in males, but not females}

Due to headcap failures, only 5 rats from each sex were able to be recorded following being trained to drink alcohol. A linear mixed effect model indicated that hormone status significantly impacted alcohol intake levels $[F(3$, $17.32)=4.11, p<0.05]$, with males drinking significantly less alcohol than females in diestrus $(p=0.052 ; 5 \mathrm{~A})$. During proestrus and estrus, female drinking amounts were not significantly different than male drinking amounts ( $p=0.073$ for proestrus; $p=0.702$ for estrus).

We also evaluated whether we could predict biological sex in the context of alcohol drinking by using LFP oscillations collected during alcohol consumption. Figure 5b shows the predictive models for males vs. females in diestrus (random permutation $\mu=44.99 \pm 0.2 \%$, real $\mu=86.81 \pm 0.01 \%, z=$ 3.76; group permutation $\mu=86.55 \pm 0.0008$ ) while alcohol was available. Again, corticostriatal oscillations do not contain more information regarding biological sex (in the context of alcohol drinking) than information about all possible groupings of rats balanced for sex.

Notably, models built from corticostriatal LFPs to predict alcohol intake levels were able to outperform randomly permuted data in males (random permutation error $=0.11 \pm 0.005$, real error $=0.03 \pm 0.001, p<0.01$; $4 \mathrm{C}$ ), but not in females (random permutation error = $0.37 \pm 0.03$, real error $=0.24 \pm 0.01, p=0.45 ; 4 \mathrm{D})$. Table 1 lists the top five neural features important in predicting alcohol naïve males vs. females in diestrus, as well as the amount of alcohol males consumed.

\section{Discussion}

Here, we demonstrate that LFP oscillations recorded within corticostriatal circuits contain significant information regarding alcohol intake levels in males, but not in females. We also show that while corticostriatal LFPs may contain some trait-level information (i.e., biological sex), the amount of information is similar to that observed in group permutations of animals balanced for sex. In the females, we observed small fluctuations in model accuracies as a function of ovarian hormone status, which correlated with observed differences in alcohol intake across phases of estrous and between sexes. Overall, the current experiment suggests that inherent electrical activity within corticostriatal circuits is not substantially different between sexes, but that the neural circuits that contain information regarding alcohol intake are sexually dimorphic.

The most compelling data from this study is that corticostriatal oscillations predicted alcohol intake levels in males, but not in females. When single feature logistic regression models were applied to each neural feature, we determined that low-frequency power in the NAcSh (particularly in the $\theta, \alpha$, and $\beta$ ranges) was negatively associated with alcohol intake levels in males. Interestingly, NAcSh $\theta$ power, while negatively correlated with alcohol intake in males, also tended to be higher in males compared to females in diestrus (when males were drinking significantly lower amounts of alcohol compared to females). While these data are correlative, they do suggest that NAcSh $\theta$ power might represent a trait-level neural feature that relates to the sex differences observed in alcohol consumption. Previous studies have demonstrated that $\theta$ oscillations in the striatum, which are coherent with hippocampal rhythms, are implicated in working memory and attention tasks, and are inhibited by NAc dopamine receptor blockade [35-37]. Along with the present study, these findings collectively suggest that NAc $\theta$ oscillations may be important in reward learning, and that low-frequency NAcSh oscillations may perhaps serve as a potential therapeutic target in future research.

This work is further supported by previous studies using corticostriatal oscillations to characterize the neurobiological underpinnings of alcohol-drinking behaviors in male rats. For instance, in male rats chronically exposed to alcohol, $\beta$ power in the NAcSh is reduced during alcohol-consumption periods compared to alcohol-deprivation periods [21]. This change in NAcSh $\beta$ power coincides with an increase in NAcSh dopamine content, suggesting that changes in NAcSh $\beta$ oscillations are influenced by dopamine signaling in the

Table 1 Neural features important in model prediction accuracies

\begin{tabular}{|c|c|c|c|c|c|}
\hline \multicolumn{3}{|l|}{ Male vs. female (diestrus) } & \multicolumn{3}{|c|}{ Predicting alcohol intake levels: males } \\
\hline Feature & Mean AUC & Direction & Feature & $R^{2}$ & Slope \\
\hline Left NAcSh $\theta$ & 0.818 & Male $>$ Female & Right NAcSh a & 0.505 & -243.08 \\
\hline Right NAcSh $\theta$ & 0.788 & Male $<$ Female & Left NAcSh a & 0.483 & -283.80 \\
\hline Left mPFC-right NAcSh I $\gamma$ & 0.782 & Male $<$ Female & Left NAcSh $\theta$ & 0.422 & -186.40 \\
\hline Left mPFC-right NAcSh hy & 0.767 & Male $<$ Female & Right mPFC - Right NAcSh IY & 0.409 & -4.37 \\
\hline Left mPFC-left NAcSh $\Delta$ & 0.766 & Male > Female & Right NAcSh $\beta$ & 0.393 & -82.98 \\
\hline
\end{tabular}

The top 5 LFP features used in models predicting males vs. diestrus females and alcohol intake levels in males. Frequency bands [delta $(\Delta)$, theta $(\theta)$, alpha $(a)$, beta $(\beta)$, low gamma (l $\gamma)$, and high gamma (hy)] are described for power features within and coherence features between neural sites 
striatum (or vice-versa). Additionally, alcohol-preferring $\mathrm{P}$ rats (sex unspecified) show reduced PFC-NAc $\theta$ coherence, which is enhanced during alcohol drinking, compared to Wistar rats, suggesting that reduced connectivity in corticostriatal circuits may be related to the increased alcohol consumption in P rats [26]. A significant amount of future work is required to understand the neural circuit dynamics of reward behavior across rat strains and across spatial resolutions (e.g., from single-cell to multi-cell to LFP recordings), but the current data supports the notion that electrical signals recorded in the NAcSh can serve as a valuable readout of substance use behaviors in male rodents.

There are several potential circuits that may contain more information regarding alcohol intake levels in females. In clinical samples, women tend to use alcohol for negative reinforcement reasons, while men tend to use alcohol for positive reinforcement reasons [38]. Women are also more sensitive to stress-induced relapse [5, 39], and similar results have been seen in rodent models of alcohol drinking, where female rats are more sensitive to stressinduced reinstatement of alcohol seeking [40]. Therefore, regions involved in emotional regulation may contain more information about female drinking behavior. One particular region of interest is the insula, which is activated by natural and drug rewards, is involved in craving, and integrates emotional stimuli contributing to mood regulation [14]. Clinical studies report that reduced insular gray matter volume is correlated with increased alcohol expectancy in female problem drinkers, but not in male problem drinkers [41]. Interestingly, insular activation is further enhanced by alcohol cues in alcohol-dependent women compared to non-dependent women, while men show greater alcohol cue reactivity in the striatum compared to women [42, 43]. In light of these previous reports, the current experiment supports the notion that different neural circuits regulate alcohol-drinking behaviors in males and females. Ongoing work from our group aims to elucidate what brain regions contain information about female alcohol intake, focusing on the anterior insula and amygdala.

The current findings align well with previous work describing sex differences in alcohol-drinking behavior. Here, we replicate findings that female rats (in diestrus) drink more alcohol than male rats when accounting for body weight, with female alcohol intake levels fluctuating slightly across the different phases of estrous [12, 13, 44]. Interestingly, when predicting phase of estrous in females from corticostriatal LFPs, the accuracies of the prediction models line up with differences in drinking levels across estrous phases. Specifically, the model predicting estrus from diestrus performed the best, which aligns with the phases in which female drinking behavior is most different. These data are particularly interesting considering that ovarian hormone status has been shown to influence addictive behavior in female rats and in women (though less so with alcohol and more so with other addictive substances like cocaine $[5,45,46])$. Our future work will continue to investigate the role of ovarian hormones in altering substance use behaviors (and the underlying neural circuits) with the aim of developing a more comprehensive picture of the neurobiology of addiction in female rodents.

It is important to consider a couple of caveats to the current study. When attempting to predict males vs. females in diestrus, the real model outperformed models built on random permutations (chance); however, the group permutation models had a similar accuracy to the real model. This adds a layer of complexity to the interpretation of the data, as the accuracy of the group permutations suggests that the information in the circuit regarding biological sex is no greater than the information describing natural variability in circuit activity between similar sized groups of animals (balanced for sex). There are likely many psychological domains in which corticostriatal circuit activity contains information; thus, some of the group permutations may be finding real differences between rats that are not related to biological sex. However, if biological sex was associated with substantially different corticostriatal oscillations, we would expect the real models to perform better than both the random and group permutations. It is unclear whether adding more rats to the experiment would have altered the relative accuracies of the real models and group permutations, so our future work will systematically analyze how many animals/samples are necessary to build a group permutation model with accuracies that approach chance. Nevertheless, this limitation does not reduce the importance of the present data. The models predicting alcohol intake levels in males and females were withinanimal, meaning that the neural features identified in the continuous prediction models are directly related to the variability in alcohol intake in males.

Secondly, Sprague-Dawley rats tend to drink less alcohol than other rodent strains [44], and the male rats in this study were indeed drinking very small amounts of alcohol with low variability compared to the females. This variability in alcohol drinking is an important factor in interpreting model performance (error in the prediction). We would expect that models operating at chance (permuted models) predicting data with higher mean values and variance (female data) would produce greater prediction errors compared to permuted models trying to predict data with lower means and variance (male data). Because the error of the permuted models accounts for these differences in the distributions of the male and female drinking data, we can interpret the real model error by the relationship to the distribution of permuted error. Therefore, even though the male drinking data had a lower mean and 
variance, the fact that the real model could predict individual alcohol intake values with a significantly lower error than the permuted models, indicates that these models were performing better than would be expected by chance-unlike the female models. Thus, we were able to conclude that there was significant information contained within corticostriatal LFPs regarding alcohol intake in males (but not females).

Our future work aims to extend these findings to alcohol-dependent rodents (which will lead to substantial increases in alcohol intake) and other rodent strains. The goal of future work is to provide a causal link by specifically manipulating the neural features associated with alcohol intake in the hopes of changing alcoholdrinking behavior.

\section{Perspectives and significance}

The current dataset contributes to our long-term goal of characterizing the neural circuits that underlie alcoholdrinking behavior in males and females, and our data suggest that these circuits are sexually dimorphic in nature. Moreover, the present data set reinforces the need to develop more personalized therapies for alcohol-related problems, and to help achieve this aim, current work in our laboratory attempts to identify the neural circuits that underlie female alcohol-drinking behavior. Additionally, we aim to characterize how circuit oscillations change across states of alcohol dependence in males and females in order to isolate (perhaps sex-specific) neural targets for reducing problematic alcohol use.

\section{Acknowledgements}

The authors would like to thank Elise Bragg for her assistance in collecting portions of the behavioral data.

\section{Authors' contributions}

AMH and EDKS contributed to planning the experimental design, collected the, data and wrote the manuscript. LLD analyzed the local field potential data, performed the computational modeling, and contributed to editing the manuscript. KMK and EDA assisted with data collection. WTD and AIG contributed to planning the experimental design and editing the manuscript. All authors read and approved the final manuscript.

\section{Funding}

This work was supported by funds from the Department of Psychiatry at the Geisel School of Medicine at Dartmouth (AG), the Hitchcock Foundation (AH), NIDA T32 training grant (DA037202; AH), an LRP grant from the NIH NCATS (WD), an NIAAA training grant (F31AA027441; LD), and the Dartmouth Clinical and Translational Science Institute from the NIH NCATS (KL2TR001088; WD).

\section{Availability of data and materials}

The datasets used and/or analyzed during the current study are available from the corresponding author on reasonable request. This data is available as a preprint on bioRxiv (doi: https://doi.org/10.1101/750711).

\section{Ethics approval and consent to participate}

All experiments were carried out in accordance with the National Institute of Health Guide for the Care and Use of Laboratory Animals (NIH Publications No. 80-23) and were approved by the Institutional Animal Care and Use Committee of Dartmouth College.
Consent for publication

Not applicable

\section{Competing interests}

Over the past 3 years, AG has received research grants from Alkermes, Novartis, and Janssen. He has served as an (uncompensated) consultant to Otsuka and Alkermes and as a member of a Data Monitoring Board for Lilly. The other authors declare that they have no competing interests.

\section{Author details}

'Department of Psychiatry, Geisel School of Medicine at Dartmouth, Medical Center Drive, Lebanon NH 03756, USA. ${ }^{2}$ Dartmouth College, Hanover, USA.

${ }^{3}$ The Dartmouth Clinical and Translational Science Institute, Dartmouth College, Hanover, USA.

Received: 30 August 2019 Accepted: 5 December 2019

Published online: 18 December 2019

\section{References}

1. World Health Organization. Global status report on alcohol and health 2018; 2018. p. 450.

2. Erol A, Karpyak VM. Sex and gender-related differences in alcohol use and its consequences: contemporary knowledge and future research considerations. Drug Alcohol Depend. 2015;156:1-13 Available from: http:// www.ncbi.nlm.nih.gov/pubmed/26371405. Cited 21 Feb 2016.

3. Rehm J, Dawson D, Frick U, Gmel G, Roerecke M, Shield KD, et al. Burden of disease associated with alcohol use disorders in the United States. Alcohol Clin Exp Res. 2014;38(4):1068-77 Available from: http://www.pubmedcentral.nih. gov/articlerender.fcgi?artid=4147870\&tool=pmcentrez\&rendertype=abstract. Cited 16 Feb 2016.

4. Grant BF, Chou SP, Saha TD, Pickering RP, Kerridge BT, Ruan WJ, et al. Prevalence of 12-month alcohol use, high-risk drinking, and DSM-IV alcohol use disorder in the United States, 2001-2002 to 2012-2013. JAMA Psychiatry. 2017;20852:2001-2.

5. Becker JB, McClellan ML, Reed BG. Sex differences, gender and addiction. J Neurosci Res. 2017;95(1-2):136-47 Available from: http://www.ncbi.nlm.nih. gov/pubmed/27870394. Cited 21 Dec 2018.

6. Becker JB, Perry AN, Westenbroek C. Sex differences in the neural mechanisms mediating addiction: a new synthesis and hypothesis. Biol Sex Differ. 2012;3(14):1-35.

7. Beery AK, Zucker I. Sex bias in neuroscience and biomedical research. Neurosci Biobehav Rev. 2011;35(3):565-72 Available from: http://www. pubmedcentral.nih.gov/articlerender.fcgi?artid=3008499\&tool= pmcentrez\&rendertype=abstract. Cited 17 Feb 2016.

8. Zucker I, Beery AK. Males still dominate animal studies. Nature. 2010;465(7299):-690 Available from: http//www.ncbi.nlm.nih.gov/pubmed/20535186. Cited 20 Dec 2015.

9. Li T-K, Lumeng L. Alcohol preference and voluntary alcohol intakes of inbred rat strains and the National Institutes of Health heterogeneous stock of rats. Alcohol Clin Exp Res. 1984;8(5):485-6.

10. Torres OV, Walker EM, Beas BA, O'Dell LE. Female rats display enhanced rewarding effects of ethanol that are hormone dependent. Alcohol Clin Exp Res. 2014;38(31):108-15.

11. Almeida OFX, Shoaib M, Deicke J, Fischer D, Darwish MH, Patchev VK Gender differences in ethanol preference and ingestion in rats. J Clin Invest. 1998;101(12):2677-85.

12. Roberts AJ, Smith AD, Weiss F, Rivier C, Koob GF. Estrous cycle effects on operant responding for ethanol in female rats. Alcohol Clin Exp Res. 1998; 22(7):1564-9 Available from: http://www.ncbi.nlm.nih.gov/pubmed/9802543. Cited 18 Feb 2016.

13. Henricks AM, Berger AL, Lugo JM, Baxter-Potter LN, Bieniasz KV, Craft RM, et al. Sex differences in alcohol consumption and alterations in nucleus accumbens endocannabinoid mRNA in alcohol-dependent rats. Neuroscience. 2016;335:195-206

14. Koob GF, Volkow ND. Neurobiology of addiction: a neurocircuitry analysis. Lancet Psychiatry. 2016;3(8):760-73 Available from: http://www.ncbi.nlm.nih. gov/pubmed/27475769. Cited 21 Dec 2018.

15. Goto Y, Grace AA. Limbic and cortical information processing in the nucleus accumbens. Trends Neurosci. 2008;31(11):552-8 Available from: http:// linkinghub.elsevier.com/retrieve/pii/S0166223608001884. Cited 2 Apr 2018.

16. Koob GF, Volkow ND. Neurocircuitry of addiction. Neuropsychopharmacology. 2010;35(1):217-38. https://doi.org/10.1038/npp.2009.110. 
17. Goldstein RZ, Volkow ND. Drug addiction and its underlying neurobiological basis: neuroimaging evidence for the involvement of the frontal cortex. Am J Psychiatry. 2002;159(10):1642-52.

18. Howe MW, Atallah HE, McCool A, Gibson DJ, Graybiel AM. Habit learning is associated with major shifts in frequencies of oscillatory activity and synchronized spike firing in striatum. Proc Natl Acad Sci U S A. 2011;108(40): 16801-6 Available from: http://www.pnas.org/cgi/doi/10.1073/pnas.111315 8108. Cited 21 Dec 2018.

19. Thorn CA, Graybiel AM. Differential entrainment and learning-related dynamics of spike and local field potential activity in the sensorimotor and associative striatum. J Neurosci. 2014;34(8):2845-59 Available from: http://www.jneurosci. org/cgi/doi/10.1523/JNEUROSCl.1782-13.2014. Cited 21 Dec 2018.

20. van der Meer MAA, Kalenscher T, Lansink CS, Pennartz CMA, Berke JD, Redish AD. Integrating early results on ventral striatal gamma oscillations in the rat. Front Neurosci. 2010;4:300 Available from: http://journal.frontiersin. org/article/10.3389/fnins.2010.00300/abstract.Cited 21 Dec 2018.

21. Hadar R, Voget M, Vengeliene V, Haumesser JK, van Riesen C, Avchalumov $Y$, et al. Altered neural oscillations and elevated dopamine levels in the reward pathway during alcohol relapse. Behav Brain Res. 2017:316:131-5 Available from: https://linkinghub.elsevier.com/retrieve/pii/S01664328163 05630. Cited 21 Dec 2018

22. Berke JD. Fast oscillations in cortical-striatal networks switch frequency following rewarding events and stimulant drugs. Eur J Neurosci. 2009;30(5): 848-59 Available from: http://doi.wiley.com/10.1111/j.1460-9568.2009.06843. x. Cited 18 Dec 2018.

23. Morra J, Glick SD, Cheer JF. Cannabinoid receptors mediate methamphetamine induction of high frequency gamma oscillations in the nucleus accumbens. Neuropharmacology. 2012;63(4):565-74 Available from: https://linkinghub.elsevier. com/retrieve/pii/S0028390812001724. Cited 21 Dec 2018.

24. Dejean C, Boraud T, Le Moine C. Opiate dependence induces network state shifts in the limbic system. Neurobiol Dis. 2013;59:220-9 Available from: https:// linkinghub.elsevier.com/retrieve/pii/S0969996113002064. Cited 21 Dec 2018.

25. Tsurugizawa T, Abe Y, Le Bihan D. Water apparent diffusion coefficient correlates with gamma oscillation of local field potentials in the rat brain nucleus accumbens following alcohol injection. J Cereb Blood Flow Metab. 2017:37(9):3193-202

26. McCane AM, Ahn S, Rubchinsky LL, Janetsian-Fritz SS, Linsenbardt DN, Czachowski CL, et al. COMT inhibition alters cued-evoked oscillatory dynamics during alcohol drinking in the rat. Eneuro. 2018;5(October): ENEURO.0326-18.2018

27. Doucette WT, Khokhar JY, Green Al. Nucleus accumbens deep brain stimulation in a rat model of binge eating. Transl Psychiatry. 2015;5(12):e695.

28. Henderson MB, Green Al, Bradford PS, Chau DT, Roberts DW, Leiter JC. Deep brain stimulation of the nucleus accumbens reduces alcohol intake in alcohol-preferring rats. Neurosurg Focus. 2010;29(2):E12 Available from: http://thejns.org/doi/10.3171/2010.4.FOCUS10105. Cited 2 Apr 2018.

29. Henricks AM, Dwiel LL, Deveau NH, Simon AA, Ruiz-Jaquez MJ, Green Al, et al. Corticostriatal oscillations predict high vs. low drinkers in a rat model of limited access alcohol consumption. Front Syst Neurosci. 2019; 13(August):1-8.

30. Mccracken $C B$, Grace AA. Nucleus accumbens deep brain stimulation produces region-specific alterations in local field potential oscillations and evoked responses in vivo. J Neurosci. 2009;29(16):5354-63.

31. Catanese J, Carmichael JE, van der Meer MAA. Low- and high-gamma oscillations deviate in opposite directions from zero-phase synchrony in the limbic corticostriatal loop. J Neurophysiol. 2016;116(1):5-17.

32. Doucette WT, Dwiel L, Boyce JE, Simon AA, Khokhar JY, Green Al. Machine learning based classification of deep brain stimulation outcomes in a rat model of binge eating using ventral striatal oscillations. Front Psychiatry. 2018:9:336 Available from: https://www.frontiersin.org/article/10.3389/fpsyt.2 018.00336/full. Cited 15 Aug 2018

33. Dwiel LL, Khokhar JY, Connerney MA, Green Al, Doucette WT. Finding the balance between model complexity and performance: using ventral striatal oscillations to classify feeding behavior in rats. PLoS Comput Biol. 2019; 15(4):e1006838 Cohen KB, editor. Available from: http://www.ncbi.nlm.nih. gov/pubmed/31009448. Cited 16 July 2019.

34. Qian, J., Hastie, T., Friedman, J., Tibshirani, R. and Simon N. Glmnet in Matlab. 2013. Available from: http://www.stanford.edu/ hastie/glmnet matlab/. Cited 24 July 2019.

35. DeCoteau WE, Thorn C, Gibson DJ, Courtemanche R, Mitra P, Kubota Y, et al Learning-related coordination of striatal and hippocampal theta rhythms during acquisition of a procedural maze task. Proc Natl Acad Sci U S A. 2007;104(13):5644-9 Available from: http://www.pnas.org/cgi/doi/10.1073/ pnas.0700818104. Cited 15 July 2019.

36. DeCoteau WE, Thorn C, Gibson DJ, Courtemanche R, Mitra P, Kubota Y, et al. Oscillations of local field potentials in the rat dorsal striatum during spontaneous and instructed behaviors. J Neurophysiol. 2007;97(5):3800-5 Available from: http// www.physiology.org/doi/10.1152/jn.00108.2007. Cited 15 July 2019.

37. Matulewicz P, Kasicki S, Hunt MJ. The effect of dopamine receptor blockade in the rodent nucleus accumbens on local field potential oscillations and motor activity in response to ketamine. Brain Res. 2010;1366:226-32 Available from: https://linkinghub.elsevier.com/retrieve/pii/S00068993 0021542. Cited 15 July 2019.

38. Peltier MR, Verplaetse TL, Mineur YS, Petrakis IL, Cosgrove KP, Picciotto MR, et al. Sex differences in stress-related alcohol use. Neurobiol Stress. 2019;10: 100149 Available from: http://www.ncbi.nlm.nih.gov/pubmed/30949562. Cited 17 July 2019

39. Hudson A, Stamp JA. Neuroscience and biobehavioral reviews ovarian hormones and propensity to drug relapse: a review. Neurosci Biobehav Rev. 2011;35(3):427-36

40. Bertholomey ML, Nagarajan V, Torregrossa MM. Sex differences in reinstatement of alcohol seeking in response to cues and yohimbine in rats with and without a history of adolescent corticosterone exposure. Psychopharmacology (Berl). 2016;233(12):2277-87 Available from: http://link. springer.com/10.1007/s00213-016-4278-x. Cited 17 July 2019.

41. Ide JS, Zhornitsky S, Hu S, Zhang S, Krystal JH, Li CR. Sex differences in the interacting roles of impulsivity and positive alcohol expectancy in problem drinking: a structural brain imaging study. Neurolmage Clin. 2017;14:750-9 Available from: http://www.ncbi.nlm.nih.gov/pubmed/28413777. Cited 15 July 2019.

42. Tapert SF, Brown GG, Baratta MV, Brown SA. fMRI BOLD response to alcohol stimuli in alcohol dependent young women. Addict Behav. 2004;29(1):33-50 Available from: http://www.ncbi.nlm.nih.gov/pubmed/14667419. Cited 17 July 2019.

43. Kaag AM, Wiers RW, de Vries TJ, Pattij T, Goudriaan AE. Striatal alcohol cue-reactivity is stronger in male than female problem drinkers. Eur J Neurosci. 2018; Available from: http:/www.ncbi.nlm.nih.gov/pubmed/29888821. Cited 17 July 2019.

44. Priddy BM, Carmack SA, Thomas LC, Vendruscolo JCM, Koob GF, Vendruscolo LF. Sex, strain, and estrous cycle influences on alcohol drinking in rats. Pharmacol Biochem Behav. 2017;152:61-7 Available from: http:// www.ncbi.nlm.nih.gov/pubmed/27498303. Cited 21 Dec 2018.

45. Jackson LR, Robinson TE, Becker JB. Sex differences and hormonal influences on acquisition of cocaine self-administration in rats. Neuropsychopharmacology. 2006;31:129-38.

46. Allen AM, Lunos S, Heishman SJ, al'Absi M, Hatsukami D, Allen SS. Subjective response to nicotine by menstrual phase. Addict Behav. 2015;43:50-3 Available from: http://www.ncbi.n/m.nih.gov/pubmed/25553511. Cited 17 July 2019.

\section{Publisher's Note}

Springer Nature remains neutral with regard to jurisdictional claims in published maps and institutional affiliations.
Ready to submit your research? Choose BMC and benefit from:

- fast, convenient online submission

- thorough peer review by experienced researchers in your field

- rapid publication on acceptance

- support for research data, including large and complex data types

- gold Open Access which fosters wider collaboration and increased citations

- maximum visibility for your research: over $100 \mathrm{M}$ website views per year

At BMC, research is always in progress.

Learn more biomedcentral.com/submissions 